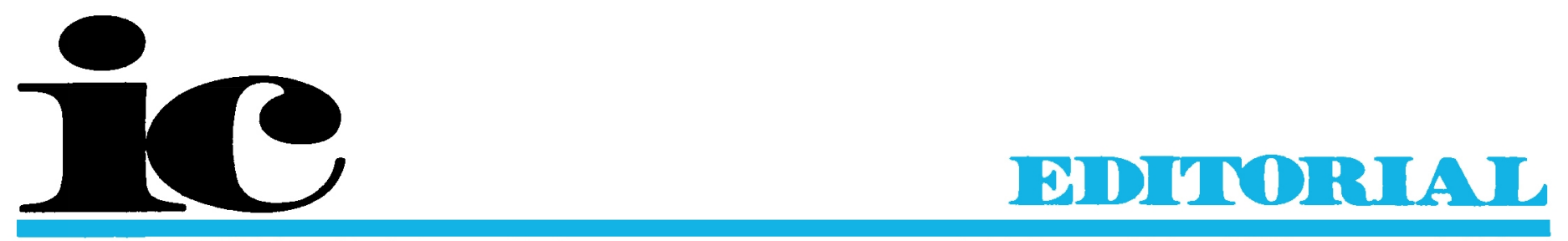

\title{
Hospital Infections
} from the WHO Perspective

There is an increasing awareness of the importance of the suffering and financial cost caused by nosocomial infections. The World Health Organization (WHO) has not been in the forefront of activities in this field. For a long time it has held the role of a passive observer of many national activities, unlike the US Centers for Disease Control or the Council of Europe. For many years these groups have been instrumental in initiating national studies. WHO became more active only in the mid-1970s.

Thousands of papers on nosocomial infections are published yearly. Most European countries have hospital infection surveillance committees and many have laws, guidelines, and surveillance systems, some on a computerized nationwide scale. Excellent practical systems and methods have been developed or tested in disinfection, cleaning, isolation, and care procedures. Meaningful prevalence data have accumulated, control measures have been devised and, above all, the cost has been reduced. However, the fact remains that these efforts have met with varying degrees of success or even failure. It has become increasingly obvious that the microorganisms in the inanimate hospital environment contribute negligibly to endemic nosocomial infections. ${ }^{1-3}$ The American Hospital Association stated that routine environmental sampling of the hospital air, floors, and objects has not contributed significantly to the prevention of nosocomial. infections. ${ }^{4}$ Responsible organisms appear to be derived more often from the human body (including hospital employees and visitors) rather than the inanimate environment. ${ }^{5}$

Nosocomial infections are considered a worldwide problem, affecting both the developed and developing countries. These infections occur in both epidemic and endemic forms and are among the major causes of morbidity and mortality in hospitalized patients, leading directly or indirectly to the emergence of new health

Address reprint requests to Univ. Prof. Boris Velimirovic, Chief, Communicable Diseases, World Health Organization, Regional office for Europe, Scherfigsvej 8, DK-2100 Copenhagen O, Denmark. hazards for the community and becoming an increasing problem for health authorities. If this is not yet realized by health authorities everywhere, it is because hospital infections do not appear in notification lists and are not statistically counted as infections.

Twenty years ago Dubos warned:

The point at issue is that the microbial diseases which account for the greatest morbidity in our communities today are completely different in their origin and manifestation from those which are so effectively dealt with by modern techniques ... The sciences concerned with microbial diseases have developed almost exclusively from the study of acute or semi-acute infectious processes caused by virulent microorganisms acquired through exposure to an exogenous source of infection. In contrast, the microbial diseases most common in our communities today arise from the activities of microorganisms that are ubiquitous in the environment, persist in the body without causing any harm under ordinary circumstances, and exert pathological effects only when the infected person is under conditions of physiological stress. ${ }^{6}$

This has introduced a new challenge which has become even greater since the appearance of plasmid-mediated antibiotic resistance. But plasmid analysis also brings opportunities to distinguish between two otherwise similar bacterial strains and to determine their sources and reservoirs and transmission mechanisms. ${ }^{7}$

What has emerged over the years can be summarized as follows:

1. The problem of nosocomial infection cannot be separated from: a) antibiotic therapy or resistance of microorganisms, b) the immunosuppression studies in identification of high risk persons, and c) molecular biology and immunology research.

2. The problem of nosocomial infection and of antibiotic resistance, whatever the source of infection (endogenous, exogenous or cross-infection) is an explicit epidemiological problem requiring the joint attention of many disciplines including clinicians, microbiologists, epidemiologists, infection control workers and public health administrators. 
Both facts contradict the formerly, and sometimes still held opinion that hospital infections and antibiotic resistance are problems of the hospitals alone. While this may be valid for incidence in a particular local hospital, prevalence of infection is the concern of the scientific medical community, the entire country, and even demands international responsibility.

In recent years the risk of serious bacterial, mycotic and viral infections has actually increased among many groups of patients in the developed countries, particularly those with surgical prostheses (from hip joints to artificial heart valves), burn patients, transplant recipients, those with immunosuppression due either to disease or cytotoxic therapy, patients in intensive care units, the aged and neonates. ${ }^{8}$ The WHO has found that prima facie comparison and simple extrapolation are impossible without standardization of methods and protocols, and due attention being paid to differences in the type of hospitals, patients, prevalent pathogens and prophylactic and management practices.

It is also apparent that the beneficial results of antibiotic therapy are being progressively eroded by the emergence of increasing numbers of antibiotic-resistant bacteria. From the data available to WHO, it seems that this is happening in all countries of the world, just as hospital infections are occurring everywhere. But thus far, no coordinated program has been implemented to estimate the extent of the problem on a global scale. There is no validation regarding significant regional differences in the prevalence of bacteria resistant to different antibiotics but the data do indicate a significantly higher incidence of antibiotic resistance in countries where aminoglycosides are used outside the institutional setting. It is not known how rapidly such resistance is appearing.

Continuing ignorance of such trends impairs protection against infections at all levels. Health authorities are unable to make the most effective use of the finances, manpower and other resources at their disposal. The pharmaceutical industry faces unnecessary uncertainties and handicaps in the expensive and lengthy process of developing new antibiotics. Physicians, who are often obliged to select initial antibiotic therapy on clinical grounds alone, may choose one to which the infecting organism is resistant and thus increase the patient's chances of either prolonged illness or death from bacterial infections. ${ }^{8}$

The Scientific Working Groups of the WHO considened the problems of hospital infections and antibiotic resistance in 1981 and 1982.8 .9 They concluded that a surveillance program that could effectively gather and collate information about the similarities and differences in the epidemiological nature of hospital infection and antibiotic resistance among the bacterial flora of the general population would be of great assistance, especially in forecasting future trends. To this effect, common definitions were adopted at a meeting in Brussels, Belgium. ${ }^{10}$

Each day many thousands of laboratories worldwide isolate bacteria from patients with hospital infections and test the bacteria's susceptibility to antibiotics. The rich source of existing information is presently used only as a guide to therapy for the individual patient. The informa- tion about the sale of antibiotics provides an immediately available data base for the surveillance program.

New understanding of the biology of antibiotic resistance now makes it increasingly possible to interpret the results of the surveillance program at many levels. The first level is the traditional and still most pragmatic one of observing the actual resistance, measured in isolates, which hampers the treatment of individual patients. At another level, however, is the epidemiological follow-up of the dynamics of host/microorganism interaction at the molecular level. We know now that most antibiotic resistance can be accounted for by 30 or 40 antibiotic-resistant genes, and that in most centers some ten multiply-resistant genes may account for most of the resistance encountered. The antibiotic-resistant genes are most often mediated by plasmids, may be transposed from one plasmid to another within a cell, transferred from one bacterial cell to another, or carried in a bacterial cell which leaves one patient and colonizes another. In this way, and largely propelled by selection pressure of antibiotic usage (assumed to be strongest in hospitals), individual antibiotic-resistant plasmids distribute and equilibrate through the complex interconnecting bacterial isosystems which are carried by every animal. Every resistant isolate in every patient may be seen as part of some epidemic. It is now increasingly possible to trace resistant plasmid spread by observing the distribution of distinctive antibiotypes in surveillance data and confirming plasmid identity in a few representative isolates using DNA molecular fingerprinting techniques. ${ }^{8}$

An essential part of the surveillance program would be the continuing revision and improvement of existing analytical programs and the development and integration of new ones to accommodate newly perceived needs and opportunities. Methods of analysis in both hospital infections and antibiotic surveillance follow two general directions. One direction leads toward data compression, the goal being to produce summarizing overviews of differences or trends.

Other methods of analysis run in the opposite direction toward the expansion and elaboration of detailed data useful for delineating a local problem or a perceived epidemic.

It is increasingly recognized that healthy people may be part of the chain of transmission of antibiotic-resistant bacteria. For this reason it is also desirable for laboratories to study the resistance patterns of organisms obtained from people not directly seeking medical attention. Such studies would require careful epidemiological planning to take into account inevitably difficult sampling from urban, rural and possibly migratory population groups.

The experts advising the WHO emphasized that a comprehensive nosocomial infection control and prevention program, adaptable to all countries, is an appropriate goal for the WHO. Following the recommendations of the technical groups on proposals for defining the problem of measurement, a plan for a collaborative surveillance survey has been outlined to determine the scale, common factors and differences of nosocomial infections in various regions of the world. ${ }^{8-13}$

In 1983 the $\mathrm{WHO}$ is initiating a large program of 
collaborative surveillance of hospital infections and antibiotic resistance with all countries of the world. The main objectives are:

- to identify determinants and mechanisms and quantify patterns of hospital infections and assume valid comparison;

- to identify the individuals and population at greatest risk;

- to evaluate the benefit of different methods used in the prevention, management, surveillance and control of nosocomial infections, including their cost effectiveness.

The proposed program will allow a country to enter at a point appropriate to its own current level of involvement and available resources. As a first step a WHO Collaborating Centre for reference and research on hospital infections was established in 1981 at the Division of Hospital Infections, PH Laboratories, London, England. It is hoped that such efforts could provide valuable clues leading to possible methods to improve the presently deteriorating situation.

\section{REFERENCES}

1. McGowan JE Jr: Whence come nosocomial infections? N Engl J Med 1982; 307:1576-1577.

2. McGowan JE Jr: Environmental factors in nosocomial infection-A selective focus. Rev Infect Dis 1982; 3:760-769.

3. Maki DG, Alvardo CJ, Hassener CA, et al: Relation of the inanimate hospital environment to endemic nosocomial infection. $N$ Engl J Med 1982; 307:1562-1566.
4. Committee on Infections Within Hospitals, American Hospital Association: Statement on microbial sampling in the hospital. Hospitals 1974; 48:125-126.

5. Schaffner $W$ : Humans; the animate reservoir of nosocomial pathogens, in Cundy KR, Ball W (eds): Infection Control in Health Care Facilities: Microbiological Surveillance. Baltimore, University Park Press, 1977, pp 57-70.

6. Dubos R: Man Adapting. New Haven, Yale University Press, 1965, chap VII, p 164.

7. Parrott PL, Terry PM, Whitworth EH, et al: Pseudomonas aeruginosa peritonitis associated with contaminated polaxomeriodine solution. Lancet 1982; 2:683-685.

8. Antimicrobial Resistance-Report of a Scientific Working Group. Geneva, World Health Organization, 1981.

9. Surveillance, Control and Prevention of Hospital-Acquired (Nosocomial) Infections 1981-Report of a Scientific Group. Geneva, World Health Organization, 1981.

10. Report on a Working Group on Nosocomial Infections. Brussels, World Health Organization, 1982, Copenhagen, 1983.

11. Ducel G, Haxhe JJ, Tanner F, et al: Practical Guide to the Prevention of Hospital-Acquired Infections. Geneva, World Health Organization, 1979, Bacterial Diseases Uni. 79, Revision 1.

12. Hospital-Acquired Infections: Guidelines to Laboratory Methods. Copenhagen, World Health Organization, 1978, Reg. Publ. European Series, No. 4.

13. Public Healh Aspects of Antibiotic-Resistant Bacteria in the Environment. Report on a Consultation Meeting. Brussels, 1975, World Health Organization, Copenhagen, 1976.

Professor Dr. B. Velimirovic, MD, DIM, DTPH

Chief, Communicable Diseases Regional Office for Europe World Health Organization Denmark, Europe 